\title{
Postoperative adjuvant TACE-associated nomogram for predicting the prognosis of resectable Hepatocellular Carcinoma with portal vein Tumor Thrombus after Liver Resection
}

Fuchen Liu $^{1 *}$, Xinggang Guo ${ }^{1,2^{*}}$, Wei Dong ${ }^{1 *}$, Wenli Zhang ${ }^{1 *}$, Shuxun Wei ${ }^{3}$, Shutong Zhang ${ }^{4}$, Xiuli Zhu ${ }^{5}$, Weiping Zhou ${ }^{1}$, Jinmin Zhang ${ }^{6}$ and Hui Liu ${ }^{\circledR}$

1. The Third Department of Hepatic Surgery, Eastern Hepatobiliary Surgery Hospital, Second Military Medical University, Navy Medical University, Shanghai 200438, China.

2. Changhai Hospital, Second Military Medical University, Navy Medical University, Shanghai 200438, China.

3. The First Department of General Surgery, Changzheng Hospital, Second Military Medical University, Navy Medical University, Shanghai 200438, China.

4. Department of Nephrology, First Affiliated Hospital, Anhui Medical University, Hefei, China.

5. Department of Gastroenterology, Anhui Provincial Hospital, University of Science and Technology of China, Hefei, 230001, China.

6. Department of Anesthesiology, Eastern Hepatobiliary Surgery Hospital, the Second Military Medical University, Shanghai, China.

*These authors have contributed equally to this work.

$\square$ Corresponding authors: Hui Liu, M.D., Ph.D., The Third Department of Hepatic Surgery, Eastern Hepatobiliary Surgery Hospital, Second Military Medical University, Navy Medical University, Shanghai 200438, China. Tel: (+86)021-81875523; E-mail: liuhuigg@hotmail.com; Jinmin Zhang, M.D., Department of Anesthesiology, Eastern Hepatobiliary Surgery Hospital, the Second Military Medical University, Navy Medical University, Shanghai, 200438, China. Tel: (+86)021-81875523; E-mail: 56441962@qq.com.

(c) The author(s). This is an open access article distributed under the terms of the Creative Commons Attribution License (https://creativecommons.org/licenses/by/4.0/). See http://ivyspring.com/terms for full terms and conditions.

Received: 2020.04.10; Accepted: 2020.09.13; Published: 2020.10.23

\begin{abstract}
Background: To explore the effects of postoperative adjuvant transarterial chemoembolization (PA-TACE) on the prognosis of HCC patients with Portal Vein Tumor Thrombus (PVTT) undergoing resection, and to develop a PA-TACE-related nomogram for predicting survival individually.

Patients and Methods: Two hundred and ninety-three consecutive HCC patients with PVTT under RO hepatectomy were recruited. Forty-seven cases had recurrence within one month after surgery. The remaining 246 cases consisted of 90 PA-TACE and 156 non-PA-TACE cases. COX regression analysis was performed for overall survival (OS) or recurrence-free survival (RFS) of these 246 cases, allowing the derivation of independent factors that were integrated into the nomogram. C-index, calibration curves, and risk stratification were performed to evaluate the performance and discriminative power of the nomograms.

Results: In 246 patients without recurrence within one month after surgery, the OS and RFS for the PA-TACE group were significantly better than those for the non-PA-TACE group $(P<0.0001, P<0.0001$, respectively). After Cox regression analysis of OS or RFS, PA-TACE-related nomogram models were constructed. The C-index of the PA-TACE-related nomogram for OS and RFS was 0.72 and 0.73 , respectively. Calibration curves revealed a good agreement between predictions and observations for the nomograms. Based on the nomogram-related risk stratification, Kaplan-Meier curves showed powerful discriminative ability.

Conclusions: PA-TACE therapy improved the survival of HCC patients with PVTT undergoing hepatectomy. Accurate nomogram models were developed for predicting the individual survival and recurrence of these patients.
\end{abstract}

Key words: Postoperative Adjuvant TACE, HCC with PVTT, Hepatectomy, Nomogram, Prognosis 


\section{Introduction}

Hepatocellular carcinoma (HCC) is one of the most common malignancies. Once diagnosed, however, most HCC is terminal and has lost the chance for radical resection [1], especially in patients with portal vein tumor thrombus (PVTT) which has a high incidence of $44 \%-62.2 \%$ [2]. According to the Barcelona Clinic Liver Cancer (BCLC) guidelines, although sorafenib is the recommended treatment for HCC patients with PVTT [3], transarterial chemoembolization (TACE) and liver resection are still considered to be options $[4,5]$.

In advanced HCC with PVTT, TACE is commonly recommended for a subset of patients [6]. A previous prospective study showed that TACE for HCC patients with PVTT achieved both higher 12and 24-month overall survival rates than the conservative approach [7]. Other reports have also shown that TACE is safe and feasible compared with other conservative treatments for HCC patients with PVTT [8].

Surgical resection and liver transplantation are still regarded as the curative modalities for advanced HCC, including cases with port vein invasion [9]. The high costs and shortage of organ donors are significant barriers for liver transplantation [10]. Many previous studies have revealed that liver resection for HCC patients with PVTT has a survival benefit compared with interventional treatment and other conservative measures $[4,8,11]$. In Japan, one previous study compared 1058 surgical patients with 1058 non-surgical patients with HCC and PVTT using propensity score matching, and this study concluded that the surgical approach should be applied for selected HCC patients with tumor invasion limited to the first-order branch of the portal vein, as these patients obtained a longer survival outcome than those receiving non-surgical treatment [12]. However, the significant incidence of postoperative recurrence and metastasis was found to be a critical challenge for surgical therapy in HCC with PVTT $[13,14]$.

Nowadays, treatment strategies for HCC with PVTT remain controversial [15]. Various combination therapies, including TACE, radiotherapy, and sorafenib, are commonly used for HCC patients with PVTT, especially TACE treatment $[15,16]$. Our previous study developed a novel and stable nomogram model for precise individualized therapy for hepatitis B virus (HBV)-related HCC with postoperative adjuvant TACE (PA-TACE). Notably, we have not seen the individual prediction model associated with PA-TACE in HCC patients with PVTT.

In this present study, we explored the influence of PA-TACE on the survival and recurrence of patients with HCC and PVTT who had undergone resection. PA-TACE was usually performed one month after the surgical resection. We aimed to construct a PA-TACE-related nomogram model for predicting the survival risk probability of these advanced HCC patients with port vein invasion who had undergone liver surgery.

\section{Methods}

\section{Patients}

HCC patients with type I/II PVTT, diagnosed according to Cheng's PVTT classification [17], who had undergone R0 radical liver resection in the Eastern Hepatobiliary Surgery Hospital (EHBH) from January 2008 to May 2013 were considered for inclusion. In the retrospective study, candidate patients were recruited based on the following inclusion criteria: (1) diagnosed with HCC pathologically; (2) no preoperative treatment; (3) Child-Pugh A and B; (4) type I/II PVTT; (5) initially underwent R0 surgical resection; (6) treated with PA-TACE one month after resection without other adjuvant treatment. The exclusion criteria were: (1) extrahepatic metastasis or lymph node metastasis; (2) incomplete clinical or follow-up data; (3) diagnosed with other malignancies. It should be noted that PVTT in HCC patients was diagnosed based on typical preoperative radiological indications (computed tomography (CT), magnetic resonance imaging (MRI)) and/or intraoperative and postoperative pathological reporting.

According to the inclusion and exclusion criteria, a total of 293 HCC patients with PVTT were enrolled in the present study. Forty-seven cases recurred within one month after liver resection, 90 cases were treated with PA-TACE one month after liver resection, and 156 cases did not receive any antitumor treatment until relapse one month after hepatectomy.

\section{Preoperative management and liver resection}

After admission, the patients underwent routine abdominal ultrasonography, chest radiography, electrocardiogram, and a pulmonary function test. Additional laboratory tests, including liver function tests, HBV or HCV-related tests, and assay for tumor markers, were also conducted. For patients with large tumors, the remnant liver volume was assessed by a three-dimensional imaging method to prevent postoperative liver failure, according to differences in their underlying liver disease.

After the preoperative safety assessment, patients who had sufficient remnant liver volume and a chance for R0 liver resection were considered candidates for hepatectomy. Anatomical or non- 
anatomical resection was performed depending on the location, extension, and size of the tumors. A clamp-crushing approach was used for separating the liver parenchyma. Hilar clamping was performed for hepatic portal occlusion depending on the intraoperative situation. R0 liver resection was performed based on an absence of residual tumor tissue and a negative microscopic surgical margin.

\section{Postoperative management and Adjuvant TACE}

Patients who had experienced no recurrence one month after resection and whose liver function had returned to normal underwent postoperative adjuvant TACE. The recurrence criteria are detailed in our previous study [18]. Using the Seldinger method, adjuvant TACE was conducted for the entire remnant liver of these postoperative patients, via the proper location of the femoral artery, under the guidance of hepatic and CT angiography. Chemotherapeutic agents, including fluorouracil, epirubicin, and platinum, and embolic agents, including lipiodol and gelatin sponge, were then placed into the proper hepatic artery through the femoral artery using a catheter. The dosage of lipiodol and doxorubicin was evaluated and determined by the body surface area and the remaining liver volume.

\section{Definitions}

Tumor rupture was diagnosed based on preoperative examinations (abdominal ultrasonography, CT, or MRI) and further confirmed by intraoperative detection. With the cutoff of 2000 $\mathrm{IU} / \mathrm{mL}, \mathrm{HBV}$-DNA load $\geq 2000 \mathrm{IU} / \mathrm{mL}$ was defined as high level, and HBV-DNA load $<2000 \mathrm{IU} / \mathrm{mL}$ was defined as low level [19]. Four hundred $\mathrm{ng} / \mathrm{ml}$ was considered as the cutoff of AFP (a-fetoprotein level) between the high and low levels [19]. A blood platelet level of $100 \times 10^{9} / \mathrm{L}$ was defined as the cutoff level [20].

Tumor differentiation was evaluated and determined according to the Edmondson-Steiner classification [21]. The definitions of microvascular invasion (MVI) and satellite lesions were as detailed in our previous studies [18, 22].

\section{Follow-up and end-points}

Considering the high recurrence rate, the patients were followed up every two weeks within the first month after resection. Serum marker tests, abdominal ultrasonography, CT, or MRI was conducted on the patients. For these patients, recurrence within one month after liver resection was considered the primary end-point. When recurrence occurred within one month after surgery, these patients underwent the other treatment modalities.
One month after surgery, a subset of the remaining patients without recurrence underwent PA-TACE treatment. In the first year after liver resection, the patients were followed up every two months, by the routine examinations (serum tumor markers, abdominal ultrasonography, CT or MRI). The secondary end-points were overall survival (OS) (from the date of surgery to patient death or last follow-up date), and recurrence-free survival (RFS), which was calculated from the date of the surgery to the date of first documented tumor recurrence or death after liver resection. And every 6 months was conducted for them subsequently, all patients were followed up until October 2016.

\section{Statistical analysis}

The baseline features of the patients were displayed as mean value (standard error, SD) for continuous variables and frequencies (percentage, \%) for categorical variables. The $\chi^{2}$ test, or the Fisher exact test, was performed to compare the categorical variables. For continuous variables with normal distribution, the $t$-test was performed, while the Mann-Whitney $U$-test was used for continuous variables with skewed distributions. The KaplanMeier method was used to depict the survival curves for OS and RFS, which was compared using the log-rank test. Based on the results of the Cox univariate regression analysis, the independent indicators with $P$-values $<0.05$ were selected into the next Cox multivariate regression analysis. The above statistical analysis was performed using SPSS software, version 25 (IBM Corp., Armonk, NY).

Independent factors derived from the Cox multivariable analysis were integrated into the nomogram model using the $R$ rms package ( $R$ version 3.5.1), where the discriminatory ability was evaluated by the C-index. The Kaplan-Meier method was used to depict the calibration curves to assess the agreement between predictions and observations. Risk group stratification (high- and low-risk groups) was based on the total points generated from the nomogram using the X-title software [23]. A two-tailed $P$-value less than 0.05 was defined as statistically significant.

\section{Results}

\section{Patients and clinical characteristics}

A total of 31 clinical features, including 13 preoperative features, 10 intraoperative and pathological factors, and 8 pre-PA-TACE factors, were enrolled into this study, as shown in Supplemental Table 1. Two hundred and ninety-three primary liver cancer patients with PVTT were classified as advanced HCC, according to the BCLC staging; of 
these, more than $90 \%$ had hepatitis B viral infections. A total of 47 cases had a short-term recurrence within one month after surgery (Supplemental Table 1, Fig. $1)$. The other 246 cases were then divided into two groups (PA-TACE $(n=90)$ vs. non-PA-TACE $(n=156))$, according to whether they received postoperative adjuvant TACE treatment (Table 1, Fig. 1). Moreover, one to three days before PA-TACE treatment, eight pre-PA-TACE clinical factors were recorded. The same clinical factors in the 156 cases not receiving PA-TACE were also recorded at corresponding times.

\section{Poor Postoperative RFS rate and OS rate of HCC patients with PVTT after liver resection}

After liver resection, the $293 \mathrm{HCC}$ patients with PVTT had a low median overall survival time of 10.05 months, while the 1-, 2-, 3-, 4-, and 5-year overall survival rates were $40 \%, 21 \%, 14 \%, 12 \%$, and $10 \%$, respectively. In terms of cancer recurrence, the 293 cases also had a low recurrence-free time of 6.84 months, with the 1-, 2-, 3-, 4-, and 5-year recurrence- free rates were $12 \%, 8 \%, 5 \%, 5 \%$, and $4 \%$, respectively.

\section{PA-TACE benefited the survival of HCC patients with PVTT after resection}

With the exclusion of the 47 cases not suitable for PA-TACE, the two groups, the PA-TACE group $(n=90)$ and the non-PA-TACE group $(n=156)$, comprising the remaining 246 cases had significantly distinct OS and RFS rates, as depicted by KM curves (Fig. 2A-B). The PA-TACE group patients had a median overall survival time of 18.55 months, with 1-, $2-, 3-, 4-$, and 5 -year overall survival rates of $63 \%, 39 \%$, $27 \%, 22 \%$, and $18 \%$, respectively. The non-PA-TACE group patients had a median overall survival time of 9.09 months, with 1-, 2-, 3-, 4-, and 5-year overall survival rates of $34 \%, 14 \%, 10 \%, 8 \%$, and $7 \%$, respectively. At the 1-, 2-, 3-, 4-, and 5-year followups, the overall survival rates for patients of PATACE group were significantly higher than those for patients in the non-PA-TACE group (all $P^{\prime}$ s $<0.001$ ).

Table 1. Basal clinicopathological characteristics of $246 \mathrm{HCC}$ patients with PVTT with and without PA-TACE

\begin{tabular}{|c|c|c|c|c|}
\hline \multirow[t]{2}{*}{ Features } & \multicolumn{4}{|c|}{ Recurrence more than one month $(n=246)$} \\
\hline & Total patients $(n=246)$ & Non-PA-TACE $(n=156)$ & PA-TACE $(n=90)$ & $P$ \\
\hline \multicolumn{5}{|l|}{ Preoperative Factors } \\
\hline Age (mean (SD)) & $48.62(10.43)$ & $47.88(10.27)$ & $49.89(10.62)$ & 0.147 \\
\hline Sex (Male/Female) (\%) & $226(91.9) / 20(8.1)$ & $140(89.7) / 16(10.3)$ & $86(95.6) / 4(4.4)$ & 0.172 \\
\hline Tumor rupture (Yes/No) (\%) & $8(3.3) / 238(96.7)$ & $6(3.8) / 150(96.2)$ & $2(2.2) / 88(97.8)$ & 0.750 \\
\hline Child-pugh (A/B) (\%) & $209(85.0) / 37(15.0)$ & $141(90.4) / 15(9.6)$ & $68(75.6) / 22(24.4)$ & 0.003 \\
\hline HBsAg (Positive/Negative) (\%) & $222(90.2) / 24(9.8)$ & $144(92.3) / 12(7.7)$ & $78(86.7) / 12(13.3)$ & 0.225 \\
\hline HBV-DNA $(\geq 2000 /<2000 \mathrm{IU} / \mathrm{mL})(\%)$ & $128(52.0) / 118(48.0)$ & $84(53.8) / 72(46.2)$ & $44(48.9) / 46(51.1)$ & 0.537 \\
\hline Antiviral therapy (Yes/No) (\%) & $18(7.3) / 228$ (92.7) & $9(5.8) / 147$ (94.2) & $9(10.0) / 81(90.0)$ & 0.330 \\
\hline $\operatorname{AFP}(>400 / \leq 400, \mathrm{ng} / \mathrm{ml})(\%)$ & $167(67.9) / 79(32.1)$ & $110(70.5) / 46(29.5)$ & $57(63.3) / 33(36.7)$ & 0.308 \\
\hline Tbi (mean, $\mu \mathrm{mol} / \mathrm{L})(\mathrm{SD})$ & $16.72(24.89)$ & $18.51(30.89)$ & $13.61(5.24)$ & 0.138 \\
\hline PALB (mean, g/L) (SD) & $178.88(56.10)$ & $184.29(60.89)$ & $169.51(45.46)$ & 0.046 \\
\hline $\mathrm{Alb}($ mean, g/L) (SD) & $41.28(3.75)$ & $41.22(3.88)$ & $41.41(3.54)$ & 0.703 \\
\hline $\operatorname{PLT}\left(\geq 100 /<100^{*} 10^{9}\right)(\%)$ & $29(11.8) / 217(88.2)$ & $24(15.4) / 132(84.6)$ & $5(5.6) / 85(94.4)$ & 0.036 \\
\hline INR (mean (SD)) & $1.01(0.08)$ & $1.01(0.08)$ & $1.00(0.07)$ & 0.482 \\
\hline \multicolumn{5}{|l|}{ Intraoperative and Pathological Factors } \\
\hline Transfusion (Yes/No) (\%) & $92(37.4) / 154(62.6)$ & $71(45.5) / 85(54.5)$ & $21(23.3) / 69(76.7)$ & 0.001 \\
\hline Type of resection (anatomical/nonanatomical) (\%) & $75(30.5) / 171(69.5)$ & $45(28.8) / 111(71.2)$ & $30(33.3) / 60(66.7)$ & 0.553 \\
\hline Hilar clamping time (mean, minutes) (SD) & $20.64(11.98)$ & $20.43(13.27)$ & $21.00(9.41)$ & 0.72 \\
\hline Tumor size $(<10 / 5-10 / \leq 5 \mathrm{~cm})(\%)$ & $92(37.4) / 111(45.1) / 43(17.5)$ & $67(42.9) / 67(42.9) / 22(14.1)$ & $25(27.8) / 44(48.9) / 21(23.3)$ & 0.034 \\
\hline Tumor Number $(>1 / 1)(\%)$ & $86(35.0) / 160(65.0)$ & $55(35.3) / 101(64.7)$ & $31(34.4) / 59(65.6)$ & 1.000 \\
\hline Cirrhosis (Yes/No) (\%) & $153(62.2) / 93(37.8)$ & $103(66.0) / 53(34.0)$ & $50(55.6) / 40(44.4)$ & 0.135 \\
\hline Tumor capsule (Complete/Incomplete/Absent) (\%) & $13(5.3) / 112(45.5) / 121(49.2)$ & $9(5.8)) / 62(39.7) / 85(54.5$ & $4(4.4) / 50(55.6) / 36(40.0)$ & 0.056 \\
\hline Satellite lesions (Yes/No) (\%) & $32(13.0) / 214(87.0)$ & $21(13.5) / 135(86.5)$ & $11(12.2) / 79(87.8)$ & 0.935 \\
\hline MVI (Yes/No) (\%) & $216(87.8) / 30(12.2)$ & $142(91.0) / 14(9.0)$ & $74(82.2) / 16(17.8)$ & 0.067 \\
\hline Edmondson-Steiner grade (III-IV/II) (\%) & $33(13.4) / 213(86.6)$ & $11(7.1) / 145(92.9)$ & $22(24.4) / 68(75.6)$ & $<0.001$ \\
\hline \multicolumn{5}{|l|}{ Postoperative and prePA-TACE } \\
\hline postHBsAg (Positive/Negative) (\%) & $222(90.2) / 24(9.8)$ & $144(92.3) / 12(7.7)$ & $78(86.7) / 12(12.3)$ & 0.225 \\
\hline postHBV-DNA $(\geq 2000 /<2000 \mathrm{IU} / \mathrm{mL})(\%)$ & $101(41.1) / 145(58.9)$ & $71(45.5) / 85(54.5)$ & $30(34.4) / 60(65.6)$ & 0.083 \\
\hline postAFP $(>400 / \leq 400, \mathrm{ng} / \mathrm{ml})(\%)$ & $93(37.8) / 153(62.2)$ & $75(48.1) / 81(51.9)$ & $18(20.0) / 72(80.0)$ & $<0.001$ \\
\hline postTbi (mean (SD)) & $21.31(38.82)$ & $25.18(48.18)$ & $14.59(5.82)$ & 0.039 \\
\hline postAlb (mean (SD)) & $43.53(35.09)$ & $40.66(23.67)$ & $48.52(48.73)$ & 0.091 \\
\hline postPLT $\left(\geq 100 /<100 \times 10^{9}\right)(\%)$ & $38(15.4) / 208(84.6)$ & $25(16.0) / 131(84.0)$ & $13(14.4) / 77(85.6)$ & 0.883 \\
\hline postINR (mean (SD)) & $1.08(0.10)$ & $1.08(0.11)$ & $1.07(0.09)$ & 0.268 \\
\hline postAntiviral therapy (Yes/No) (\%) & $25(10.2) / 221(89.8)$ & $13(8.3) / 143(91.7)$ & $12(13.3) / 78(86.7)$ & 0.302 \\
\hline
\end{tabular}

Bold values indicate statistical significance $(\mathrm{P}<0.05)$. HCC, hepatocellular carcinoma; PVTT, Portal Vein Tumor Thrombus; PA-TACE, postoperative adjuvant transarterial chemoembolization; AFP, a-fetoprotein; Tbi, total bilirubin; PALB, prealbumin; Alb, albumin; PLT, blood platelet; INR, international normalized ratio; MVI, microvascular invasion. 


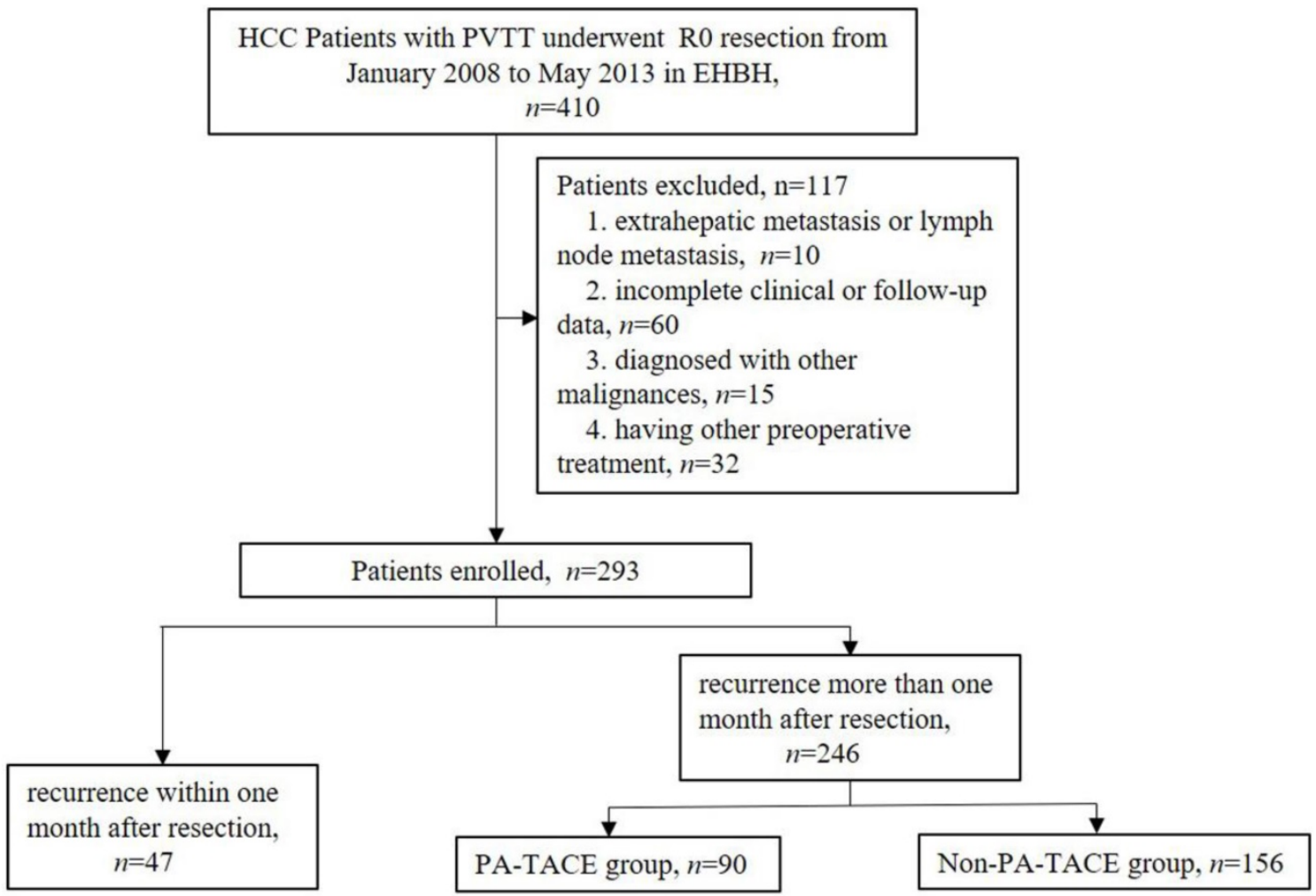

Figure 1. Flow chart showing screening and grouping of $\mathrm{HCC}$ patients with PVTT.

\section{A}

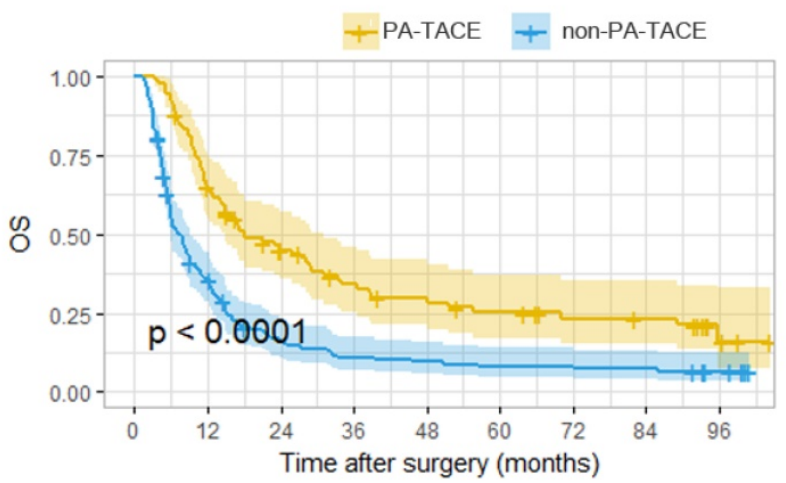

Number at risk
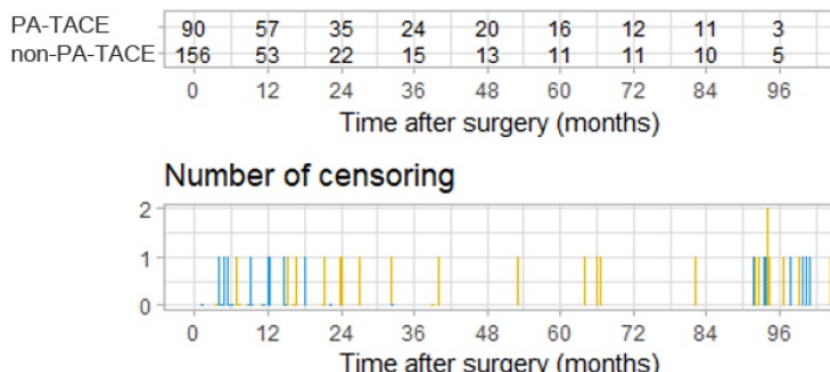

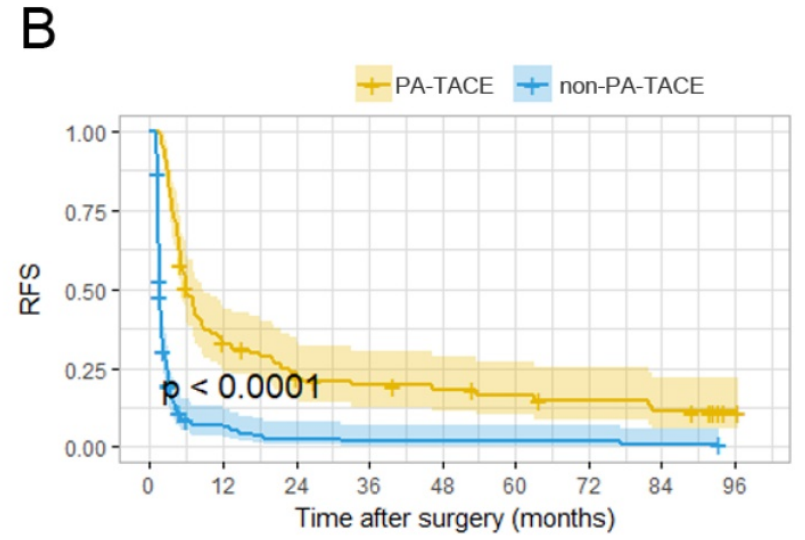

Number at risk

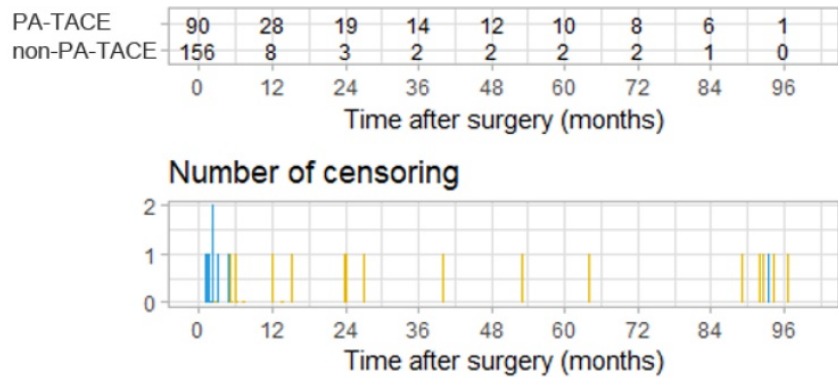

Figure 2. Kaplan-Meier analysis for predicting survival of HCC patients with PVTT with or without postoperative adjuvant TACE. A. Kaplan-Meier analysis for OS, B. Kaplan-Meier analysis for RFS.

In terms of tumor recurrence, the PA-TACE group patients had a recurrence-free time of 8.71 months, with 1-, 2-, 3-, 4-, and 5-year recurrence-free rates of $31 \%, 21 \%, 16 \%, 13 \%$, and $11 \%$, respectively.
Patients in the non-PA-TACE group had a median overall survival time of 6.32 months, with 1-, 2-, 3-, 4-, and 5 -year recurrence-free rates of $5 \%, 2 \%, 1 \%, 1 \%$, and $1 \%$, respectively. At the 1-, 2-, 3-, 4-, and 5-year 
follow-ups, the recurrence-free rates for patients of PA-TACE group were significantly higher than those for patients in the non-PA-TACE group $(P<0.001$, $P<0.010, P<0.01, P<0.001$ and $P<0.001$, respectively). These results show that PA-TACE benefited the survival of the patients with HCC and PVTT after resection not only in the OS rate but also in the RFS rate.

According to the Clavien-Dindo classification [24] (Supplemental Table 2), side effects of 90 patients who underwent adjuvant TACE were displayed. Most of the complications of PA-TACE belonged to Clavien-Dindo grade I $(86.7 \%)$, including fever, abdominal pain, nausea, and vomiting. Two $(2.2 \%)$ patients experienced grade II complications and needed blood transfusions. Only one patient $(1.1 \%$, grade III) underwent abdominal puncture drainage due to massive ascites. No one died from complications of PA-TACE in these patients.

\section{Independent risk factors for PA-TACE-related RFS and OS of PVTT patients after resection}

A total of 90 HCC patients with PVTT underwent PA-TACE therapy one month after hepatectomy and had a better survival rate than the 156 cases without PA-TACE. For PA-TACE related OS, Cox univariate regression analysis revealed that a total of seven risk factors were screened and integrated into further multivariate analysis (Table 2). Further Cox regression analysis identified five clinical features as independent risk factors (Table 3). For PA-TACE related RFS, Cox univariate regression analysis revealed that 10 risk factors were screened and integrated into further multivariate analysis (Table 2), the results of which identified three independent risk factors (Table 3). PA-TACE was also a critical independent risk feature for both OS and

A

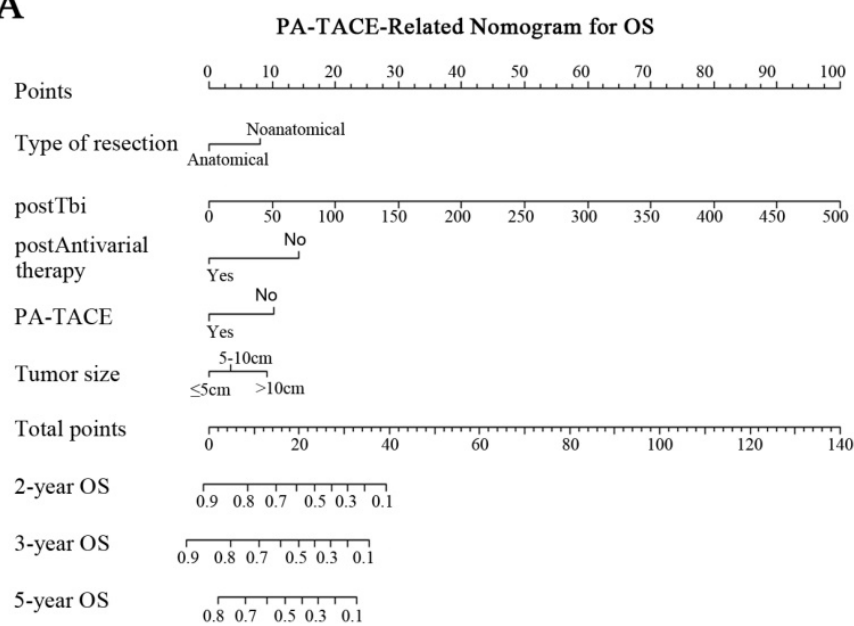

RFS of HCC patients with PVTT who had undergone liver resection and PA-TACE. Notably, the HBV DNA load and postoperative antiviral therapy benefited the survival of these groups of patients. Moreover, there is no significant difference between type I and type II PVTT in both OS and RFS of these 90 patients based on the results of Kaplan-Meier analysis (Figure S1).

\section{Construction of PA-TACE-related RFS or OS Nomogram in Predicting Survival}

According to the multivariate analysis of PATACE-related OS in the 246 cases, five independent risk factors were integrated into the PA-TACE-related nomogram model: type of resection, tumor size, posttotal bilirubin (Tbi), post-antiviral therapy, and PATACE. After undergoing resection and PA-TACE, patients who had anatomical hepatectomy or postoperative antiviral treatment had higher OS rates. However, larger tumor size, higher Tbi level, or no PA-TACE were related to a lower OS rate (Fig. 3A).

In the PA-TACE-related RFS nomogram model of 246 cases, three independent risk factors were retained: tumor number, post-HBV-DNA, and PA-TACE. Three indicators, including presence of multiple tumors, and higher postoperative HBV-DNA load or without PA-TACE, were associated with a poorer prognosis of RFS (Fig. 3B).

\section{The performance of PA-TACE-related Nomograms in Predicting Survival}

The C-index of the PA-TACE-related OS and RFS nomograms showed good discriminative ability, with values of 0.72 (95\% CI: 0.70-0.75) and 0.73 (95\% CI: 0.70-0.76), respectively. Furthermore, the calibration curves demonstrated good agreement between the predictions and the observations of 2-, 3-, and 5-year OS (Fig. 4A-C) or RFS (Fig. 4D-F), respectively.

B

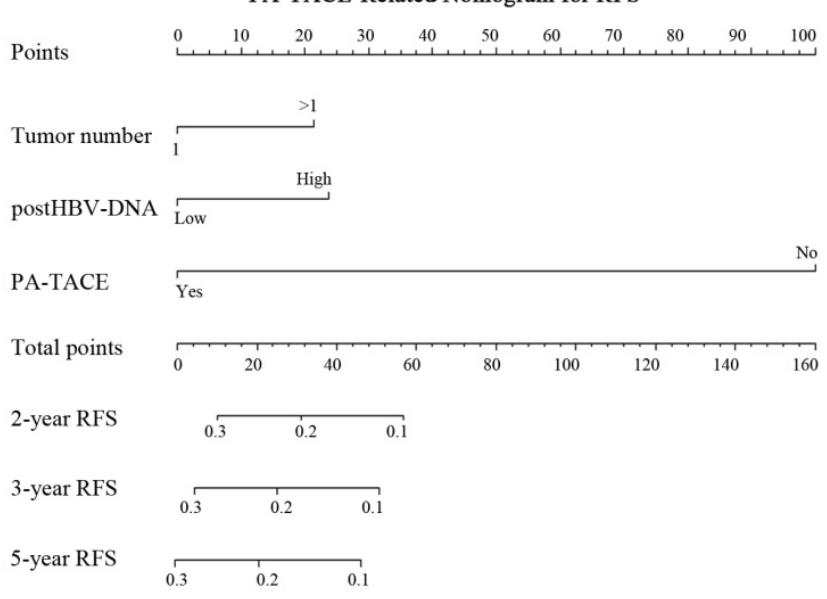

Figure 3. Nomograms for survival of HCC patients with PVTT after liver resection. PA-TACE-related nomogram for OS (A) or RFS (B). 


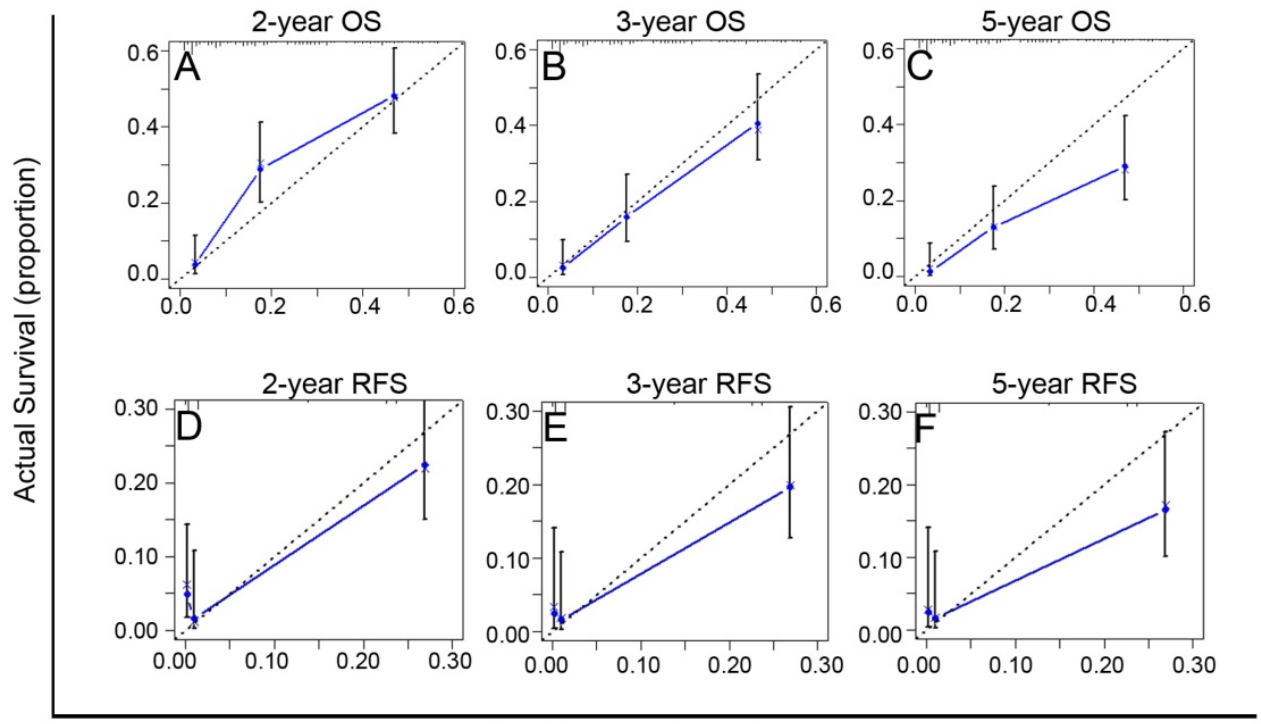

Probability predicted by Nomogram

Figure 4. Calibration curves of the PA-TACE-related nomogram models for predicting survival in 246 patients with recurrence more than one month after surgical resection. A-C. The calibration curves of the PA-TACE-related nomogram model for predicting OS at 2 years (A), 3 years (B) and 5 years (C); D-F. The calibration curves of the PA-TACE-related nomogram model for predicting RFS at 2 years (D), 3 years $(E)$ and 5 years $(F)$. $X$-axis represents nomogram-predicted probability of survival; $Y$-axis represents actual survival.

Table 2. Univariate Cox-regression analysis for predicting OS and RFS in 246 HCC patients with PVTT with and without PA-TACE

\begin{tabular}{|c|c|c|c|c|c|c|}
\hline \multirow[t]{2}{*}{ Factors } & \multicolumn{3}{|l|}{ OS } & \multicolumn{3}{|l|}{ RFS } \\
\hline & HR & $95 \% \mathrm{CI}$ & $P$ & HR & $95 \% \mathrm{CI}$ & $P$ \\
\hline \multicolumn{7}{|l|}{ Univariate analysis } \\
\hline Age (mean (SD), Years) & 1.000 & $0.99-1.02$ & 0.584 & 0.990 & $0.98-1$ & 0.096 \\
\hline Sex (Male/Female) & 1.370 & $0.83-2.25$ & 0.214 & 1.270 & $0.79-2.03$ & 0.327 \\
\hline Tumor Rupture (Yes/No) & 1.220 & $0.57-2.61$ & 0.600 & 1.320 & $0.65-2.67$ & 0.448 \\
\hline Child-pugh (A/B) & 0.800 & $0.54-1.18$ & 0.254 & 0.520 & $0.35-0.76$ & 0.001 \\
\hline HBsAg (Positive/Negative) & 0.950 & $0.6-1.49$ & 0.826 & 1.360 & $0.88-2.12$ & 0.167 \\
\hline HBV-DNA $(\geq 2000 /<2000 \mathrm{IU} / \mathrm{mL})$ & 1.220 & $0.93-1.61$ & 0.154 & 1.210 & $0.93-1.58$ & 0.158 \\
\hline Antiviral therapy (Yes/No) & 0.640 & $0.36-1.14$ & 0.131 & 0.860 & $0.52-1.43$ & 0.566 \\
\hline $\operatorname{AFP}(>400 / \leq 400 \mathrm{ng} / \mathrm{ml})$ & 1.360 & $1.01-1.84$ & 0.043 & 1.340 & $1.01-1.79$ & 0.044 \\
\hline Tbi (mean, $\mu \mathrm{mol} / \mathrm{L})$ & 1.000 & $1-1.01$ & 0.069 & 1.000 & $1-1.01$ & 0.272 \\
\hline PALB (mean, g/L) & 1.000 & $1-1.00$ & 0.634 & 1.000 & $1-1.00$ & 0.211 \\
\hline $\mathrm{Alb}($ mean, g/L) & 1.000 & $0.96-1.04$ & 0.926 & 0.970 & $0.93-1$ & 0.068 \\
\hline PLT $\left(\geq 100 /<100 \times 10^{9}\right)$ & 1.100 & $0.73-1.68$ & 0.649 & 1.320 & $0.89-1.97$ & 0.168 \\
\hline INR (mean) & 3.040 & $0.49-18.83$ & 0.231 & 3.140 & $0.51-19.35$ & 0.217 \\
\hline Transfusion (Yes/No) & 1.410 & $1.07-1.87$ & 0.016 & 1.270 & $0.97-1.68$ & 0.088 \\
\hline Type of resection (anatomical/nonanatomical) & 0.590 & $0.43-0.8$ & 0.001 & 0.870 & $0.65-1.16$ & 0.328 \\
\hline Hilar clamping time (mean, minutes) & 0.990 & $0.98-1$ & 0.076 & 1.000 & $0.99-1.02$ & 0.557 \\
\hline Tumor size, $\leq 5 \mathrm{~cm}$ & \multicolumn{3}{|c|}{1.00 (Reference) } & \multicolumn{3}{|c|}{1.00 (Reference) } \\
\hline $5 \mathrm{~cm}-10 \mathrm{~cm}$ & 1.550 & $1.01-2.37$ & 0.044 & 1.360 & 0.93-1.99 & 0.111 \\
\hline$>5 \mathrm{~cm}$ & 2.290 & $1.49-3.51$ & $<0.001$ & 1.760 & $1.19-2.61$ & 0.004 \\
\hline Tumor Number $(>1 / 1)$ & 1.110 & $0.83-1.47$ & 0.495 & 1.330 & $1.01-1.75$ & 0.042 \\
\hline Cirrhosis (Yes/No) & 1.090 & $0.82-1.44$ & 0.567 & 1.110 & $0.85-1.46$ & 0.449 \\
\hline Tumor capsule, Complete & \multicolumn{3}{|c|}{1.00 (Reference) } & \multicolumn{3}{|c|}{1.00 (Reference) } \\
\hline Incomplete & 0.840 & $0.63-1.11$ & 0.223 & 0.670 & $0.5-0.88$ & 0.004 \\
\hline Absent & 1.330 & $0.72-2.49$ & 0.364 & 0.930 & $0.51-1.7$ & 0.081 \\
\hline Satellite lesions (Yes/No) & 1.340 & $0.9-1.98$ & 0.148 & 1.360 & $0.93-1.99$ & 0.113 \\
\hline MVI (Yes/No) & 1.810 & $1.15-2.85$ & 0.011 & 1.940 & $1.24-3.03$ & 0.004 \\
\hline Edmondson-Steiner grade (III-IV/II) & 1.150 & $0.77-1.71$ & 0.501 & 2.080 & $1.38-3.14$ & $<0.001$ \\
\hline postHBsAg (Positive/Negative) & 0.950 & $0.6-1.49$ & 0.826 & 1.360 & $0.88-2.12$ & 0.167 \\
\hline postHBV-DNA( $\geq 2000 /<2000 \mathrm{IU} / \mathrm{mL})$ & 1.200 & $0.91-1.58$ & 0.207 & 1.410 & $1.08-1.84$ & 0.012 \\
\hline postAFP $(>400 / \leq 400 \mathrm{ng} / \mathrm{ml})$ & 1.720 & $1.3-2.27$ & $<0.001$ & 1.540 & $1.17-2.02$ & 0.002 \\
\hline postTbi (mean, $\mu \mathrm{mol} / \mathrm{L})$ & 1.020 & $1.01-1.02$ & $<0.001$ & 1.000 & $1-1.01$ & 0.243 \\
\hline postAlb (mean, g/L) & 1.000 & $1-1.01$ & 0.114 & 1.000 & $1-1.00$ & 0.718 \\
\hline postPLT $\left(\geq 100 /<100 \times 10^{9}\right)$ & 0.950 & $0.65-1.39$ & 0.796 & 1.280 & $0.89-1.83$ & 0.179 \\
\hline postINR (mean) & 3.330 & $0.9-12.31$ & 0.071 & 1.260 & $0.36-4.39$ & 0.717 \\
\hline postAntiviral therapy (Yes/No) & 0.330 & $0.19-0.58$ & $<0.001$ & 0.890 & $0.58-1.38$ & 0.609 \\
\hline PA-TACE (No/Yes) & 2.240 & $1.67-3.02$ & $<0.001$ & 3.960 & $2.94-5.32$ & $<0.001$ \\
\hline
\end{tabular}

Bold values indicate statistical significance (P<0.05). OS, overall survival; RFS, recurrence-free survival. HCC, hepatocellular carcinoma; PVTT, Portal Vein Tumor

Thrombus; AFP, a-fetoprotein; Tbi, total bilirubin; PALB, prealbumin; Alb, albumin; PLT, blood platelet; INR, international normalized ratio; MVI, microvascular invasion. 

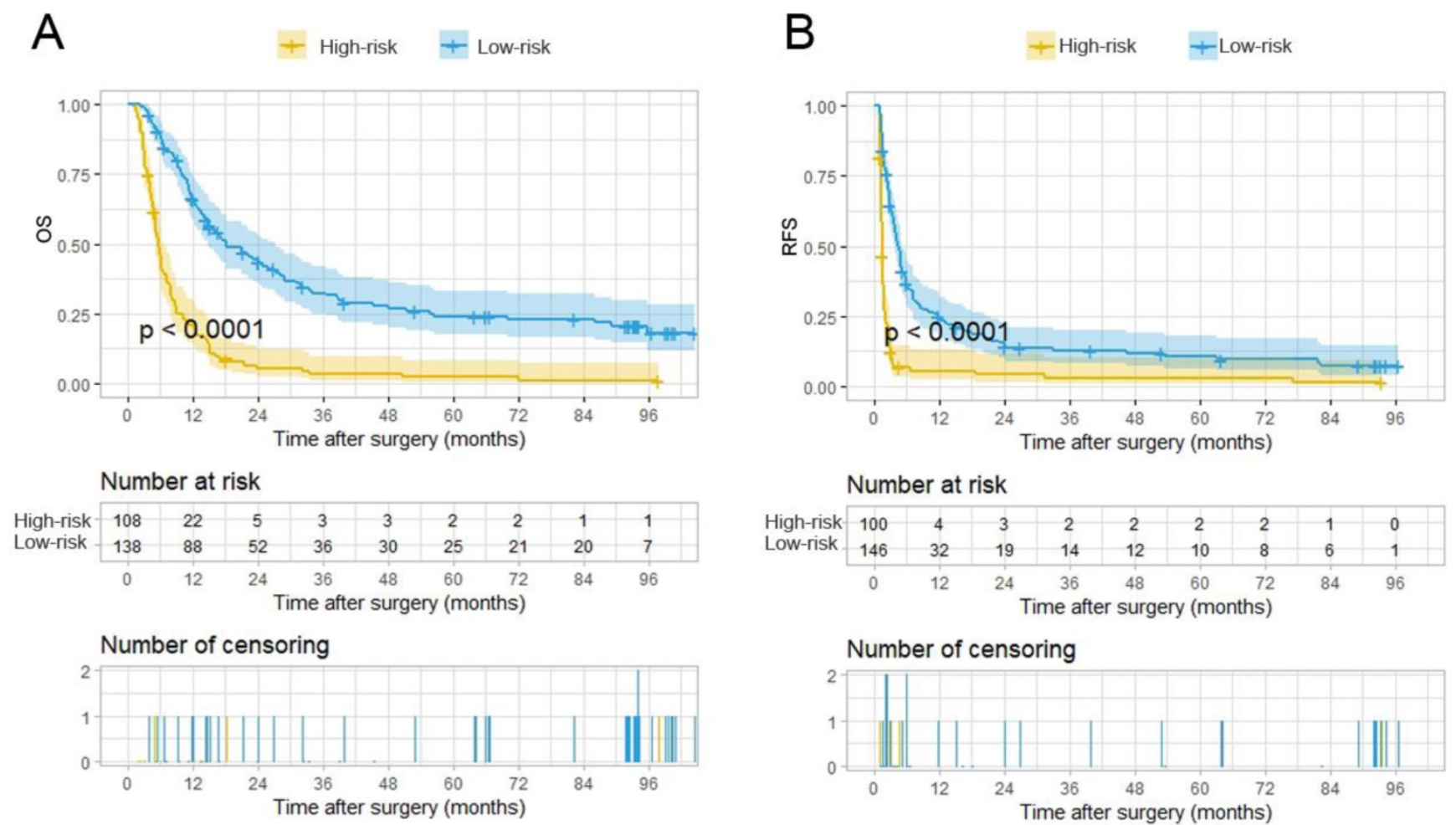

Figure 5. Kaplan-Meier analysis for survival in HCC patients with PVTT with or without PA-TACE according to the stratification risk groups (high-risk and low-risk) derived from PA-TACE-related nomogram models. A. Kaplan-Meier analysis for OS, B. Kaplan-Meier analysis for RFS.

Table 3. Multivariate Cox-regression analysis for predicting OS and RFS in 246 HCC patients with PVTT with and without PA-TACE

\begin{tabular}{|c|c|c|c|c|c|c|}
\hline \multirow[t]{2}{*}{ Factors } & \multicolumn{3}{|l|}{ OS } & \multicolumn{3}{|l|}{ RFS } \\
\hline & HR & $95 \% \mathrm{CI}$ & $P$ & HR & $95 \% \mathrm{CI}$ & $P$ \\
\hline \multicolumn{7}{|l|}{ Multivariate analysis } \\
\hline Type of resection (anatomical/nonanatomical) & 0.550 & $0.4-0.76$ & $<0.001$ & & & \\
\hline Tumor size, $\leq 5 \mathrm{~cm}$ & 1.00 (Reference) & & & & & \\
\hline $5-10 \mathrm{~cm}$ & 1.260 & $0.81-1.95$ & 0.299 & & & \\
\hline$>10 \mathrm{~cm}$ & 1.910 & $1.22-2.99$ & 0.005 & & & \\
\hline Tumor Number $(>1 / 1)$ & & & & 1.430 & $1.05-1.94$ & 0.023 \\
\hline postHBV-DNA $(\geq 2000 /<2000 \mathrm{IU} / \mathrm{mL})$ & & & & 1.330 & $1.01-1.77$ & 0.045 \\
\hline postTbi (mean, $\mu \mathrm{mol} / \mathrm{L}$ ) & 1.010 & $1.01-1.02$ & $<0.001$ & & & \\
\hline postAntiviral therapy (Yes/No) & 0.340 & $0.19-0.6$ & $<0.001$ & & & \\
\hline PA-TACE (No/Yes) & 2.06 & $1.49-2.85$ & $<0.001$ & 3.79 & $2.74-5.23$ & $<0.001$ \\
\hline
\end{tabular}

Bold values indicate statistical significance $(P<0.05)$. OS, overall survival; RFS, recurrence-free survival. HCC, hepatocellular carcinoma; PVTT, Portal Vein Tumor Thrombus; Tbi, total bilirubin.

The total points of the 246 patients ranged between 1.64-133.22 and 0-145.16, according to the PA-TACE-related OS and RFS nomograms. The 246 cases were further classified into a high-risk group and a low-risk group by their corresponding optimal cutoff value for OS or RFS (35.7 for OS, 111.7 for RFS). Based on the risk grouping method, KM curves of OS and RFS were developed, which showed distinct differences between the high- and low-risk groups $(P<0.0001$ for OS, $P<0.0001$ for RFS) (Fig. 5A-B).

\section{Discussion}

Although Sorafenib or lenvatinib are recommended as the first-line therapy for PVTT by many guidelines for advanced HCC treatment $[3,25$, 26], recent studies have shown that surgical resection produced better survival outcomes than nonsurgical therapy in HCC patients with PVTT limited to a first-order branch of the main portal vein (MPV) or above [2, 27]. Although various novel treatment modalities, including immunotherapy, combination therapy, and interferon, have been introduced in clinics to improve the prognosis of HCC with PVTT after undergoing resection [15, 28], these efforts appear to be insufficient due to high postoperative recurrence and short-term survival time [14, 29]. Furthermore, the standard treatment for these HCC patients with PVTT remains controversial. In our study, we showed that PA-TACE improved the prognosis of HCC patients with PVTT who had undergone resection. In addition, we developed PATACE-related nomogram models for predicting the 
survival risk probability of patients with HCC and PVTT.

There has been no effective prediction model for HCC patients with PVTT after resection, especially for PA-TACE-associated cases. Although one study reported a model incorporating PA-TACE for HCC patients with PVTT, it only focused on the outcome of long-term survival time, without exploring the effects of PA-TACE on the recurrence [6]. A continuous therapy course, surgical resection and PA-TACE treatment should be considered for HCC patients with PVTT undergoing liver resection. PA-TACE treatment has relatively few complications, although, after adjuvant TACE treatment, HCC patients who had undergone curative liver resection experienced somewhat greater levels of fever, abdominal pain, nausea, and vomiting. We constructed a PA-TACErelated model for postoperative patients suitable for PA-TACE one month after resection, which integrated not only the hepatectomy-associated clinical indicators but also HBV-related factors and liver function indicators before PA-TACE treatment. The PA-TACE-related nomogram models showed good discriminative ability in predicting the prognostic risk probability for these patients. Therefore, the importance of follow-up indicators should be recognized and expanded in HCC patients with PVTT undergoing resection, as these are of great significance for guiding the follow-up and the design of postoperative therapy.

Previous PVTT-related studies only focused on the recurrence and survival differences between PA-TACE and no PA-TACE treatment in HCC patients with PVTT after resection [8]. Few reports have paid much attention to the independent risk factors for PA-TACE-related prognosis for these patients. Our study not only demonstrated that PA-TACE could significantly improve the prognosis of HCC with PVTT after surgery, but also analyzed the clinical factors, including preoperative factors, intraoperative and pathological factors, and PA-TACE-related clinical factors one month after liver resection. However, follow-up concerns of postoperative factors have not attracted much interest in previous studies.

The PA-TACE-related model showed that both postoperative antiviral therapy and lower HBV-DNA load benefited the survival of patients with HCC and PVTT who had undergone resection. A previous study from our institution reported that the postoperative antiviral modality reduced the recurrence risk in HCC patients, and improved the long-term survival [30]. Therefore, the postoperative HBV-DNA level of HBV-infected patients should be treated with greater caution and given aggressive antiviral treatment. Thus, postoperative antiviral treatment could also apply to the special group of HCC patients with PVTT who undergo resection. Total bilirubin is a critical component of liver failure staging systems [31-33], which also impaired the prognosis of HCC patients [34, 35]. Total bilirubin also integrated into the established PA-TACE-related nomogram model for predicting the risk of OS, indicating that liver function should be given sufficient attention and evaluated before PA-TACE for the HCC patients with PVTT who underwent hepatectomy. Bilirubin was also one critical component of the EHBH/PVTT Scoring System for aiding the decision making on surgical resection for HCC patients with PVTT. Moreover, for PA-TACE-related prognosis, both the numbers of tumors and their diameters have been recognized as critical independent risk factors for poor HCC prognosis $[22,36]$, which was also further confirmed in HCC patients with PVTT by previous studies $[13,36]$. Our study demonstrated that tumor size reduced the overall survival time and multiple tumors impaired the RFS of these patients.

In terms of the type of resection, the roles of anatomical and non-anatomical hepatectomy approaches in the prognosis of HCC patients are controversial [37, 38]. In our study, anatomical hepatectomy had a better postoperative long-term survival time for the HCC patients with PVTT compared with the non-anatomical hepatectomy approach, and anatomic hepatectomy should be preferred for these patients.

For resectable HCC patients with PVTT after liver resection, PA-TACE could be a priority selection, especially the patients with non-anatomical resection, multiple tumor number, larger tumor size, higher post-Tbi level or higher post-HBV-DNA load. However, this study has two limitations. Firstly, the results of our study need to be validated by multicenter cohorts, and prospective studies are also needed. Secondly, due to the higher sensitivity of digital subtraction angiography (DSA) compared to CT or MRI in the detection of tumor lesions, recurrence could not be detected earlier in the non-PA-TACE group patients one month after resection, resulting in a poorer prognosis than the patients with adjuvant TACE.

Moreover, two recent studies seemed to achieve better overall survival in unresectable HCC treated with lenvatinib plus pembrolizumab[39] (median overall survival, 22 months), or atezolizumab plus bevacizumab[40] (overall survival at 12 months, $67.2 \%$ ), compared with the prognosis of PA-TACE group patients after resection in our study (median overall survival, 18.55 months and overall survival at 12 months, 63\%). Although the above results should 
be further explored via future rigorous randomized controlled study, these studies revealed that the targeted and immunological therapy, or their combination with PA-TACE have shown strong vitality and development potential for advanced HCC treatment.

In conclusion, PA-TACE benefited the survival of HCC patients with PVTT undergoing liver resection. Nomogram models were developed and showed good discriminative ability and accuracy for predicting the sequential prognostic risk probabilities. Postoperative antiviral treatment is essential for HBV-infected patients, who should be given more frequent follow-ups and further aggressive therapy.

\section{Supplementary Material}

Supplementary figures and tables.

http://www.ijbs.com/v16p3210s1.pdf

\section{Acknowledgments}

\section{Authors' contributions}

Hui Liu and Jinmin Zhang designed the study. Fuchen Liu, Xinggang Guo, Wei Dong and Wenli Zhang analyzed results and wrote the manuscript. Fuchen Liu, Xinggang Guo, Wei Dong, Wenli Zhang, Shuxun Wei, Shutong Zhang, Xiuli Zhu and Weiping Zhou supervised clinicopathological data. Fuchen Liu, Xinggang Guo, Wei Dong and Wenli Zhang revised the text of the article. Financial support: Hui Liu and Jinmin Zhang.

\section{Ethical Statement}

The present study was approved by the Institutional Ethics Committee of the Eastern Hepatobiliary Surgery Hospital. The written informed consent was also obtained from all patients.

\section{Funding Support}

This work was supported by the National Natural Science Foundation of China (81772529, 81970453), Shanghai Science and Technology Innovation Action Plan project (19441904700), the State Key Project of China (2017YFA0504503), International Science and Technology Cooperation Program of the Ministry of Science and Technology (2011DFA32980), National Key Basic Research Program of China 973 Program (2012CB526706).

\section{Competing Interests}

The authors have declared that no competing interest exists.

\section{References}

1. Forner A, Reig M, Bruix J. Hepatocellular carcinoma. Lancet (London, England). 2018; 391: 1301-14.
2. Zhang XP, Gao YZ, Chen ZH, Chen MS, Li LQ, Wen TF, et al. An Eastern Hepatobiliary Surgery Hospital/Portal Vein Tumor Thrombus Scoring System as an Aid to Decision Making on Hepatectomy for Hepatocellular Carcinoma Patients With Portal Vein Tumor Thrombus: A Multicenter Study. Hepatology (Baltimore, Md). 2019; 69: 2076-90.

3. EASL Clinical Practice Guidelines: Management of hepatocellular carcinoma. Journal of hepatology. 2018; 69: 182-236.

4. Zhang XP, Wang K, Li N, Zhong CQ, Wei XB, Cheng YQ, et al. Survival benefit of hepatic resection versus transarterial chemoembolization for hepatocellular carcinoma with portal vein tumor thrombus: a systematic review and meta-analysis. BMC cancer. 2017; 17: 902.

5. Li XS, Wang JH, Yang XZ, Ma L, Shi YX, Song Y, et al. Beneficial effects of Cripto-1 for transarterial chemoembolization in hepatocellular carcinoma. Aging. 2019; 11: 2998-3011.

6. Liu S, Guo L, Li H, Zhang B, Sun J, Zhou C, et al. Postoperative Adjuvant Trans-Arterial Chemoembolization for Patients with Hepatocellular Carcinoma and Portal Vein Tumor Thrombus. Annals of surgical oncology. 2018; 25: 2098-104.

7. Luo J, Guo RP, Lai EC, Zhang YJ, Lau WY, Chen MS, et al. Transarterial chemoembolization for unresectable hepatocellular carcinoma with portal vein tumor thrombosis: a prospective comparative study. Annals of surgical oncology. 2011; 18: 413-20.

8. Zhang YF, Shang H, Zeng XL, Ji H, Li YM, Lu HW. Postoperative adjuvant chemo (embolization) therapy for hepatocellular carcinoma with portal vein tumor thrombosis. OncoTargets and therapy. 2018; 11: 5407-17.

9. Choi HJ, Kim DG, Na GH, Hong TH, Bae SH, You YK, et al. The clinical outcomes of patients with portal vein tumor thrombi after living donor liver transplantation. Liver transplantation : official publication of the American Association for the Study of Liver Diseases and the International Liver Transplantation Society. 2017; 23: 1023-31.

10. Barbier L, Cesaretti M, Dondero F, Cauchy F, Khoy-Ear L, Aoyagi T, et al. Liver Transplantation With Older Donors: A Comparison With Younger Donors in a Context of Organ Shortage. Transplantation. 2016; 100: 2410-5.

11. Cao F, Shen L, Qi H, Xie L, Song Z, Chen S, et al. Tree-based classification system incorporating the HVTT-PVTT score for personalized management of hepatocellular carcinoma patients with macroscopic vascular invasion. Aging. 2019; 11: 9544-55.

12. Kokudo T, Hasegawa K, Matsuyama Y, Takayama T, Izumi N, Kadoya M, et al. Survival benefit of liver resection for hepatocellular carcinoma associated with portal vein invasion. Journal of hepatology. 2016; 65: 938-43.

13. Zhang XP, Chen ZH, Zhou TF, Li LQ, Chen MS, Wen TF, et al. A nomogram to predict early postoperative recurrence of hepatocellular carcinoma with portal vein tumour thrombus after R0 liver resection: A large-scale, multicenter study. European journal of surgical oncology : the journal of the European Society of Surgical Oncology and the British Association of Surgical Oncology. 2019; 45: 1644-51.

14. Zhou L, Rui JA, Wang SB, Chen SG, Qu Q. Clinicopathological predictors of poor survival and recurrence after curative resection in hepatocellular carcinoma without portal vein tumor thrombosis. Pathology oncology research : POR. 2015; 21: 131-8.

15. Liu PH, Huo TI, Miksad RA. Hepatocellular Carcinoma with Portal Vein Tumor Involvement: Best Management Strategies. Seminars in liver disease. 2018; 38: 242-51.

16. Zhang ZM, Lai EC, Zhang C, Yu HW, Liu Z, Wan BJ, et al. The strategies for treating primary hepatocellular carcinoma with portal vein tumor thrombus. International journal of surgery (London, England). 2015; 20: 8-16.

17. Cheng S, Chen M, Cai J, Sun J, Guo R, Bi X, et al. Chinese Expert Consensus on Multidisciplinary Diagnosis and Treatment of Hepatocellular Carcinoma with Portal Vein Tumor Thrombus (2018 Edition). Liver cancer. 2020; 9: 28-40.

18. Huang J, Liu FC, Li L, Yuan SX, Yang Y, Jiang BG, et al. Prognostic Nomogram for Hepatitis B Virus-related Hepatocellular Carcinoma With Adjuvant Transarterial Chemoembolization After Radical Resection. American journal of clinical oncology. 2020; 43: 20-7.

19. Li Z, Lei Z, Xia Y, Li J, Wang K, Zhang H, et al. Association of Preoperative Antiviral Treatment With Incidences of Microvascular Invasion and Early Tumor Recurrence in Hepatitis B Virus-Related Hepatocellular Carcinoma. JAMA surgery. 2018; 153: e182721.

20. Mehrabi A, Golriz M, Khajeh E, Ghamarnejad O, Probst P, Fonouni H, et al. Meta-analysis of the prognostic role of perioperative platelet count in posthepatectomy liver failure and mortality. The British journal of surgery. 2018; 105: 1254-61.

21. Colecchia A, Scaioli E, Montrone L, Vestito A, Di Biase AR, Pieri M, et al. Pre-operative liver biopsy in cirrhotic patients with early hepatocellular carcinoma represents a safe and accurate diagnostic tool for tumour grading assessment. Journal of hepatology. 2011; 54: 300-5.

22. Huang J, Liu FC, Li L, Zhou WP, Jiang BG, Pan ZY. Nomograms to predict the long-time prognosis in patients with alpha-fetoprotein negative hepatocellular carcinoma following radical resection. Cancer medicine. 2020.

23. Camp RL, Dolled-Filhart M, Rimm DL. X-tile: a new bio-informatics tool for biomarker assessment and outcome-based cut-point optimization. Clinical cancer research : an official journal of the American Association for Cancer Research. 2004; 10: 7252-9.

24. Dindo D, Demartines N, Clavien PA. Classification of surgical complications: a new proposal with evaluation in a cohort of 6336 patients and results of a survey. Annals of surgery. 2004; 240: 205-13. 
25. Xie DY, Ren ZG, Zhou J, Fan J, Gao Q. 2019 Chinese clinical guidelines for the management of hepatocellular carcinoma: updates and insights. Hepatobiliary surgery and nutrition. 2020; 9: 452-63.

26. Meyers BM, Knox J, Cosby R, Beecroft JR, Chan KKW, Coburn N, et al. Nonsurgical management of advanced hepatocellular carcinoma: a clinical practice guideline. Current oncology (Toronto, Ont). 2020; 27: e106-e14.

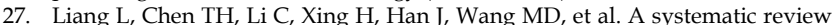
comparing outcomes of surgical resection and non-surgical treatments for patients with hepatocellular carcinoma and portal vein tumor thrombus. HPB : the official journal of the International Hepato Pancreato Biliary Association. 2018; 20: 1119-29.

28. Peng BG, He Q, Li JP, Zhou F. Adjuvant transcatheter arterial chemoembolization improves efficacy of hepatectomy for patients with hepatocellular carcinoma and portal vein tumor thrombus. American journal of surgery. 2009; 198: 313-8.

29. Glantzounis GK, Paliouras A, Stylianidi MC, Milionis H, Tzimas P, Roukos D, et al. The role of liver resection in the management of intermediate and advanced stage hepatocellular carcinoma. A systematic review. European journal of surgical oncology : the journal of the European Society of Surgical Oncology and the British Association of Surgical Oncology. 2018; 44: 195-208.

30. Huang G, Lau WY, Wang ZG, Pan ZY, Yuan SX, Shen F, et al. Antiviral therapy improves postoperative survival in patients with hepatocellular carcinoma: a randomized controlled trial. Annals of surgery. 2015; 261: 56-66.

31. Rahbari NN, Garden OJ, Padbury R, Brooke-Smith M, Crawford M, Adam R, et al. Posthepatectomy liver failure: a definition and grading by the International Study Group of Liver Surgery (ISGLS). Surgery. 2011; 149: 713-24.

32. Balzan S, Belghiti J, Farges O, Ogata S, Sauvanet A, Delefosse D, et al. The "50-50 criteria" on postoperative day 5: an accurate predictor of liver failure and death after hepatectomy. Annals of surgery. 2005; 242: 824-8, discussion $8-9$

33. Mullen JT, Ribero D, Reddy SK, Donadon M, Zorzi D, Gautam S, et al. Hepatic insufficiency and mortality in 1,059 noncirrhotic patients undergoing major hepatectomy. Journal of the American College of Surgeons. 2007; 204: 854-62; discussion 62-4.

34. Kuo $\mathrm{YH}$, Wang JH, Hung $\mathrm{CH}$, Rau $\mathrm{KM}$, Wu IP, Chen $\mathrm{CH}$, et al. Albumin-Bilirubin grade predicts prognosis of HCC patients with sorafenib use. Journal of gastroenterology and hepatology. 2017; 32: 1975-81.

35. Han S, Yang JD, Sinn DH, Ko JS, Kim JM, Shin JC, et al. Higher Bilirubin Levels of Healthy Living Liver Donors Are Associated With Lower Posttransplant Hepatocellular Carcinoma Recurrence. Transplantation. 2016; 100: $1933-8$.

36. Minagawa M, Ikai I, Matsuyama Y, Yamaoka Y, Makuuchi M. Staging of hepatocellular carcinoma: assessment of the Japanese TNM and AJCC/UICC TNM systems in a cohort of 13,772 patients in Japan. Annals of surgery. 2007; 245: 909-22.

37. Zhao H, Chen C, Gu S, Yan X, Jia W, Mao L, et al. Anatomical versus non-anatomical resection for solitary hepatocellular carcinoma without macroscopic vascular invasion: A propensity score matching analysis. Journal of gastroenterology and hepatology. 2017; 32: 870-8.

38. Marubashi S, Gotoh K, Akita H, Takahashi H, Sugimura K, Miyoshi N, et al. Analysis of Recurrence Patterns After Anatomical or Non-anatomical Resection for Hepatocellular Carcinoma. Annals of surgical oncology. 2015; 22: 2243-52.

39. Finn RS, Ikeda $M$, Zhu AX, Sung MW, Baron AD, Kudo M, et al. Phase Ib Study of Lenvatinib Plus Pembrolizumab in Patients With Unresectable Hepatocellular Carcinoma. Journal of clinical oncology : official journal of the American Society of Clinical Oncology. 2020: Jco2000808.

40. Finn RS, Qin S, Ikeda M, Galle PR, Ducreux M, Kim TY, et al. Atezolizumab plus Bevacizumab in Unresectable Hepatocellular Carcinoma. The New England journal of medicine. 2020; 382: 1894-905. 